DOI 10.17816/transsyst201843s1279-288

(C) Z. Ma ${ }^{1}$, Y. Zhao ${ }^{1}$, Y. Sun ${ }^{1}$, Z. Liao ${ }^{1,2}$, G. Lin $^{1}$

${ }^{1}$ National Maglev Transportation Engineering R\&D Center, Tongji University

${ }^{2}$ College of Electronics and Information Engineering, Tongji University

(Shanghai, China)

\title{
CONSTANT SWITCHING FREQUENCY MODEL PREDICTIVE CONTROL FOR PERMANENT MAGNET LINEAR SYNCHRONOUS MOTOR
}

Aim: This paper proposes constant switching frequency model predictive control (CSFMPC) for a permanent magnet linear synchronous motor (PMLSM) to improve the steady state and dynamic performance of the drive system.

Methods: The conventional finite control set model predictive control (FCS-MPC) can be combined with a pulse width modulation (PWM) modulator due to an effective cost function optimization algorithm which is from the idea of dichotomy. In the algorithm, all the voltage vectors in the constrained vector plane are dynamically selected and calculated through iteration. The whole system including control algorithm and mathematical model of PMLSM is built and tested by simulation using MATLAB/Simulink. Besides, the control algorithm is tested in the FPGA controller through FPGA-in-the-Loop test.

Results: With the modern digital processors or control hardware such as digital signal processors (DSPs) or field programmable gate arrays (FPGAs), the algorithm can be easily executed in less than 10-micro second. This is very proper for industrial applications. The proposed control algorithm is implemented on FPGA and tested by FPGA-in-the-Loop method. The proposed control algorithm can improve the performance of drive system greatly.

Conclusion: The proposed CSF-MPC for PMLSM not only keeps the same dynamic transient performance as FCS-MPC but also greatly decreases the torque ripple in steady state. Furthermore, CSF-MPC is also robust to parameter variations. Simulation and FPGA-in-theLoop results illustrate that CSF-MPC has an attractive performance for PMLSM drives.

Keywords: Permanent magnet linear synchronous motor (PMLSM), Model predictive control, Field programmable gate arrays (FPGAs), Dichotomy, Constant switching frequency, Voltage vector.

\section{INTRODUCTION}

Linear machines are widely used in industry systems which require fast response and large thrust. Without an intermediate conversion mechanism, a linear machine can directly convert electrical energy into mechanical energy in a linear motion [1]. This is the most attractive characteristics of linear machines. Recently, Permanent magnet linear synchronous motors (PMLSM) drive systems develop very fast because of some advantages such as low losses, fast dynamic performance and high thrust performance.

PMLSM drive systems have wide applications in industrial automation production lines and industrial transmissions such as machine tools. With the 
continuous development of equipment manufacturing technology, on the one hand, PMLSM drive system requires higher control bandwidth and dynamic response; on the other hand, it needs higher steady state accuracy and better static characteristics. These technique demand make advantage control strategies of PMLSM more desirable $[2,3]$. Recent years, with the rapid development of control platform devices, such as DSP (digital signal processor) and FPGA (field programable gate array), model predictive control (MPC) becomes attractive in control of electrical drive systems for some advantages such as good dynamic response and straightforward inclusion of nonlinearity and restrictions in the model and control $[4,5]$. For application in power electronics and electrical drives, MPC can be concluded into two categories: continuous control set MPC (CCS-MPC) and finite control set MPC (FCS-MPC).

For CCS-MPC, generalized predictive control (GPC) is a typical control strategy belong to this category [6]. GPC is established on linear models and extended to including constraints. In order to receive a required accuracy, the prediction of GPC usually need at least several steps. Moreover, the calculation is normally so large that it can only be realized by off-line calculation. Furthermore, GPC degrades accuracy while control of nonlinear systems such as electrical drive systems. FCS-MPC considers only finite set of possible switching states of power converters and solves the cost function for each of them and selects a switching state which minimizes the cost function. Easy to be implemented and good dynamic response are the two remarkable advantages of FCS-MPC. Therefore, it becomes rather popular in control of power electronic systems in these years [6, 7]. However, only one switching state to be applied in a sampling interval makes a variable switching frequency. Meanwhile, a relatively large control error is generated in each switching interval especially for two level converters. Hence, a large current ripple and wide range harmonics frequency are unavoidable.

Recently, many research works try to solve above men9tioned problems of FCS-MPC. In [8], based on a fixed hysterics bandwidth, multi-step prediction is adopted to select a proper switching state so that it keeps a constant torque ripple bandwidth. In each sampling interval, following an active switching state, a zero state will be inserted in the control interval to decrease the current ripple [9]. While in [10], a cost function for minimum torque ripple is directly defined to calculate the switching point. Based on duty cycle control, an improved model predictive control for current control is proposed in [11]. In [12], dichotomy solution algorithm for cost function is proposed which provide an effective approach to solve some of these problems.

This paper proposed a dichotomy solution based constant switching frequency model predictive control (CSF-MPC) for PMLSM. The $d$ and q axes currents are 
both predicted based on the fundamental model of PMLSM using Euler formula. The optimal voltage vector can be chosen in the whole voltage vector plane since the dichotomy solution algorithm is used. Through FPGA-in-the-Loop test, the advantages of proposed CSF-MPC compared with conventional FCS-MPC is proved. Mathematical model of PMLSM is described in the next section of this paper. Then, Section III presents conventional FCS-MPC for PMLSM. The proposed control algorithm is elaborated in Section IV. After FPGA-in-the-Loop test, we conclude the paper in Section VI.

\section{MATHEMATICAL MODEL OF PMLSM}

In this work, a simplified mathematical model of PMLSM which includes no magnetic saturation is considered. Due to the inductances of PMLSM are normally varied with position information, we mainly consider the mathematical model in synchronous rotating frame, i.e., d-q coordinate. Firstly, the d-q frame voltage equations can be described as:

$$
\begin{aligned}
& u_{d}=R_{s} i_{d}+\frac{d \lambda_{d}}{d t}-\frac{\pi v}{\tau} \lambda_{q} \\
& u_{q}=R_{s} i_{q}+\frac{d \lambda_{q}}{d t}+\frac{\pi v}{\tau} \lambda_{d}
\end{aligned}
$$

where, $u_{d}, u_{q}$ are the $\mathrm{d}$-q axis voltage; $i_{d}, i_{q}$ are the $\mathrm{d}$-q axis current; $\lambda_{d}, \lambda_{q}$ are the d-q axis flux, respectively. $R_{s}$ is the resistance of on phase PMLSM primary winding; $v$ is the moving speed of the mover; $\tau$ is the pole pitch.

The flux linkage equations in $d$-q frame can be written in the following equations:

$$
\begin{gathered}
\lambda_{d}=L_{d} i_{d}+\lambda_{f} \\
\lambda_{q}=L_{q} i_{q}
\end{gathered}
$$

where, $L_{d}, L_{q}$ are the d-q axis inductances, respectively; $\lambda_{f}$ is the flux linkage produced by permanent magnet.

Correspondingly, the electromagnetic thrust of PMLSM is deduced as the following equation:

$$
F_{e}=\frac{3 \pi}{2 \tau} n_{P}\left[\lambda_{f} i_{q}+\left(L_{d}-L_{q}\right) i_{d} i_{q}\right]
$$

For multi-pole linear motors, $n_{P}$ denotes pole-pairs.

Because the air gap is relatively large for the linear machines, the inductance value in $\mathrm{d}$ and $\mathrm{q}$ frame could be seen as the same. This means $L_{d}=L_{q}=L_{s}$. Therefore, the equation of electromagnetic thrust can be simplified as 


$$
F_{e}=\frac{3 \pi}{2 \tau} n_{P} \lambda_{f} i_{q}=k_{f} i_{q}
$$

In equation (6), $k_{f}$ is the coefficient of electromagnetic thrust.

The equation used to model the dynamics is presented in (7), where $m$ is the mass of linear movement part; $F_{l}$ is the load resistance; $D$ is the system friction coefficient.

$$
m \frac{d v}{d t}=F_{e}-F_{l}-D v
$$

\section{CONVENTIONAL FCS-MPC FOR PMLSM}

Similar with conventional direct torque control (DTC) proposed in the 1980 s which is continuously developed and improved by ABB cooperation, FCSMPC also considers finite switching states of power converters. Therefore, the optimization problem is reduced to evaluate all possible switching states and select the one which minimizes the given cost function. Based on this idea, the complex model predictive control optimization strategy of three-phase two-level inverter is simplified to optimize only eight possible switch states. Although the calculation burden will increase exponentially when the prediction horizon increases, one step prediction usually receives satisfied performance.

In the $d$-q synchronous rotating frame, the d-axis and q-axis current state space equations of PMLSM are respectively described as

$$
\frac{d i_{d}}{d t}=\frac{1}{L_{s}}\left[u_{d}-R_{s} i_{d}+\frac{\pi v}{\tau} L_{s} i_{q}\right]
$$
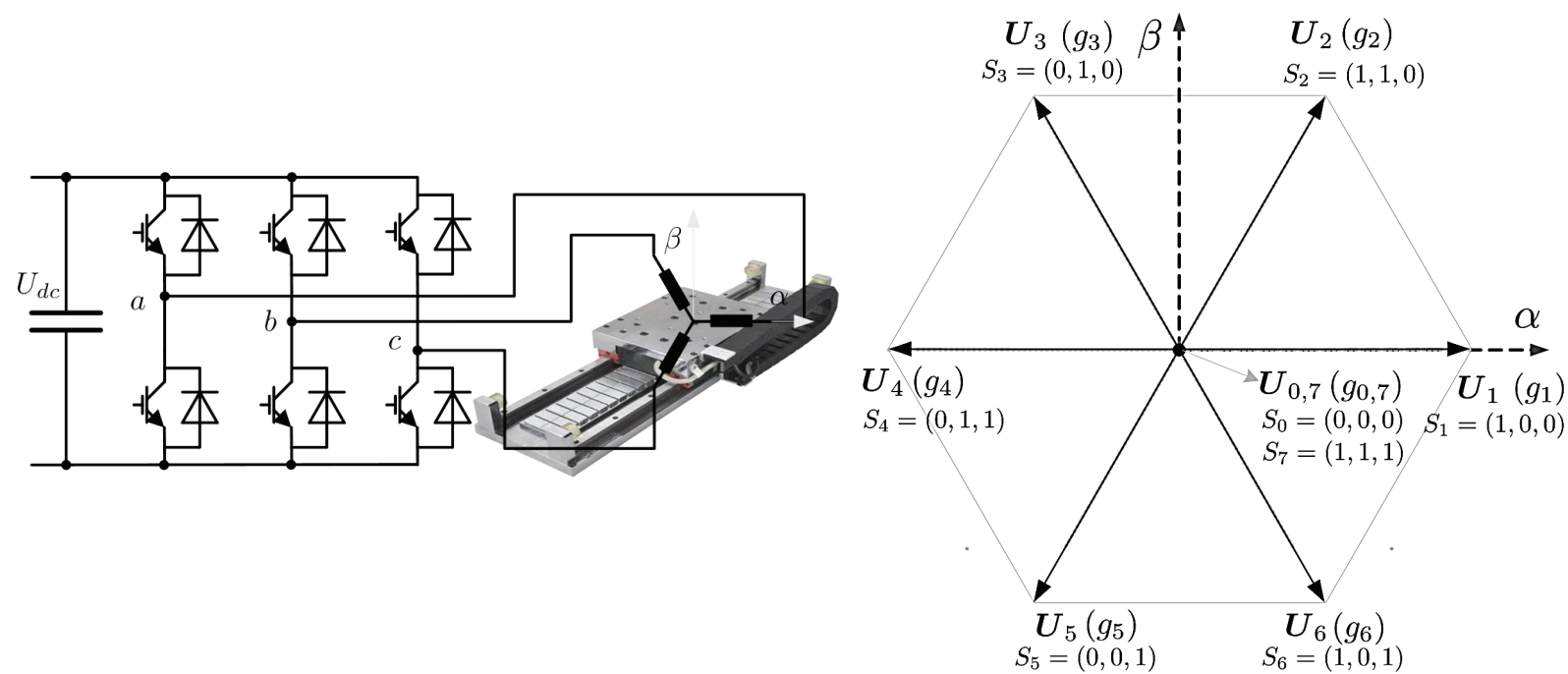

Fig. 1. PMLSM drive system: three-phase two-level inverter and its eight voltage vector state 


$$
\frac{d i_{q}}{d t}=\frac{1}{L_{s}}\left[u_{q}-R_{s} i_{q}-\frac{\pi v}{\tau} L_{s} i_{d}-\frac{\pi v}{\tau} \lambda_{f}\right]
$$

In order to calculate predicted current in the next sampling interval, Euler formula is used to discretize the current equations. This means the approximated $\mathrm{d}$-axis and q-axis current can be presented by the following equations

$$
\begin{gathered}
i_{d}(k+1)=i_{d}(k)+\frac{T_{s}}{L_{s}}\left[u_{d}(k)-R_{s} i_{d}(k)+\frac{\pi v(k)}{\tau} L_{s} i_{q}(k)\right] \\
i_{q}(k+1)=i_{q}(k)+\frac{T_{s}}{L_{s}}\left[u_{q}(k)-R_{s} i_{q}(k)-\frac{\pi v(k)}{\tau} L_{s} i_{d}(k)-\frac{\pi v(k)}{\tau} \lambda_{f}\right]
\end{gathered}
$$

Where, $i_{d}(k+1)$ and $i_{q}(k+1)$ are the predicted $\mathrm{d}$ and $\mathrm{q}$ axes current in the next sampling interval, respectively; $i_{d}(k)$ and $i_{q}(k)$ are the measured feedback $\mathrm{d}$ and $\mathrm{q}$ axes current at present sampling interval; $T_{s}$ is the sampling period; $u_{d}(k)$ and $u_{q}(k)$ are the $\mathrm{d}$ and q axes voltage, $v(k)$ is the speed all at present time.

Conventional FCS-MPC replaces the inner current control loop of fieldoriented control (FOC) by model predictive control method. The speed control loop keeps the same to use proportional integration (PI) controller. For conventional FCS-MPC, equation (10) and (11) are firstly used to calculate the one step prediction value for $\mathrm{d}$ and $\mathrm{q}$ axes current. Then, through calculation of cost function (12), the voltage vector which is able to minimize the cost function will be selected as the optimal voltage vector and applied to the inverter. Fig. 2 shows the control structure of conventional FCS-MPC for PMLSM drive system.

$$
g=\left|i_{d}{ }^{*}-i_{d}(k+1)\right|+\left|i_{q}{ }^{*}-i_{q}(k+1)\right|
$$

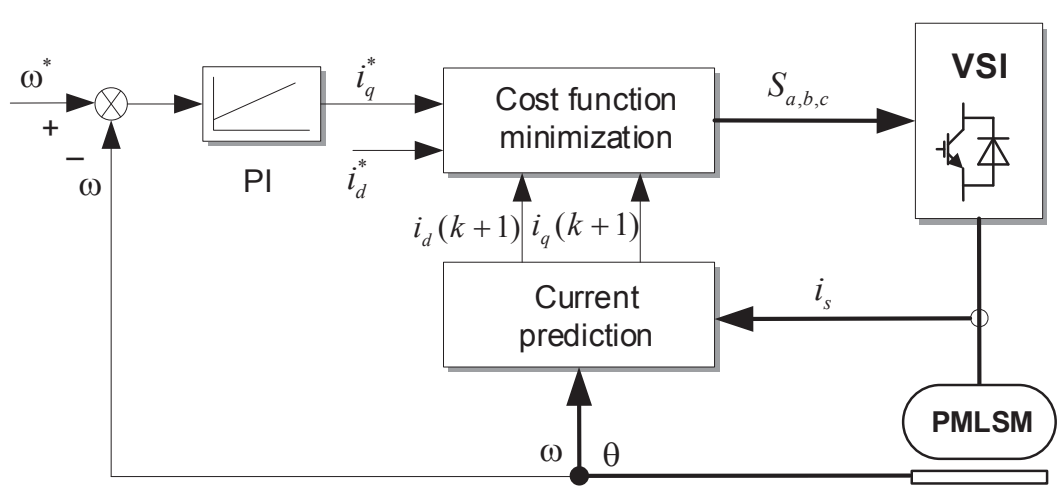

Fig. 2. Conventional FCS-MPC (predictive current control) for PMLSM drive system

\section{PROPOSED CSF-MPC FOR PMLSM}

Though the concept of FCS-MPC is straightforward and it is easy to implement this control strategy, FCS-MPC for PMLSM will present large steady 
state current and torque errors. To improve its performance, this work proposes a dichotomy solution based constant switching frequency model predictive control (CSF-MPC) for PMLSM. The structure of CSF-MPC for PMLSM drive system is presented in Fig. 3. We can see the difference between conventional FCS-MPC and proposed CSF-MPC for PMLSM drive system (Fig. 2 and Fig. 3) is PWM module is required of CSF-MPC. The reason is that the output voltage vector of CSF-MPC is quasi-continuous, so PWM module can be applied to the control system. Due to the proposed dichotomy solution algorithm, the optimal voltage vector in each sampling interval can be acquired in time. In the proposed CSF-MPC, the over current protection is added in addition,

$$
\begin{gathered}
G=\left|i_{d}^{*}-i_{d}(k+1)\right|+\left|i_{q}^{*}-i_{q}(k+1)\right|+g_{I} \\
g_{I}=\left\{\begin{array}{l}
0, \text { if } i_{s}<I_{s . \max } \\
\infty, \text { else }
\end{array}\right.
\end{gathered}
$$

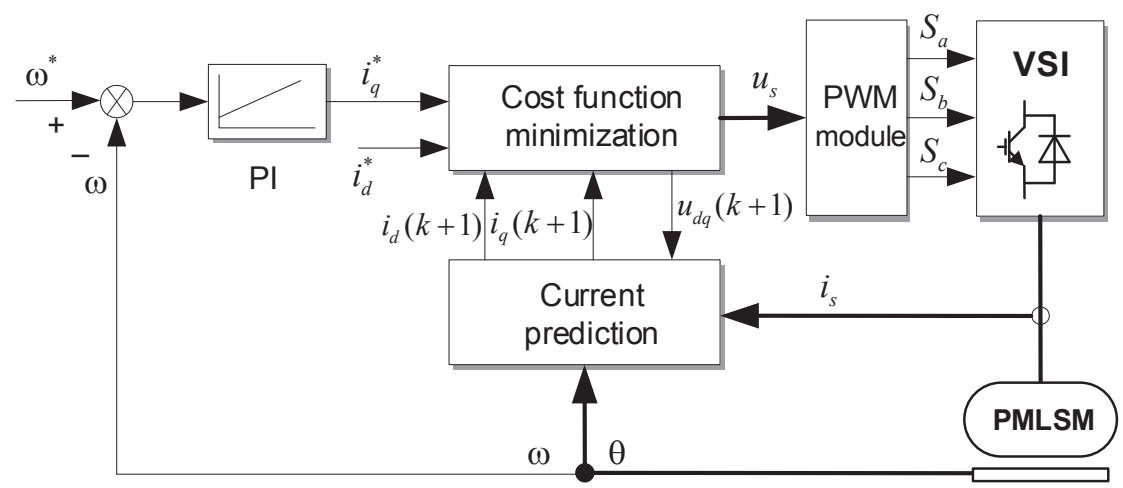

Fig. 3. Proposed CSF-MPC for PMLSM drive system

The idea of the cost function solution algorithm is from dichotomy. As presented in Fig. 4, the voltage vector search area is the voltage circular plane with a radius of $U_{m}$ - the maximum available voltage. In each sampling interval, with $U_{m} / 2$ amplitude and $\pi / 4$ phase different of each other, eight voltage vectors are selected as the vector candidates at the initialization. At the first step of the iteration, one of the eight vectors is selected as the optimal vector based on the cost function.

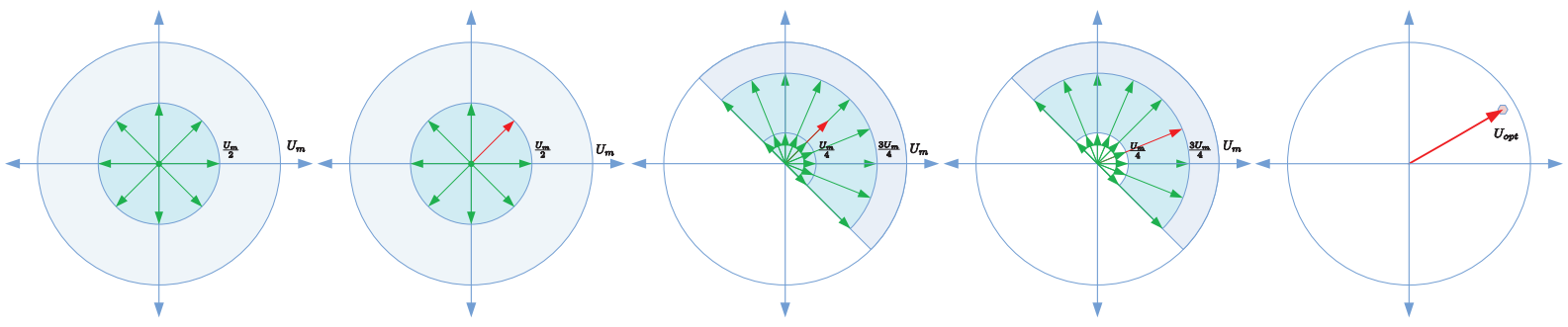

Fig. 4. Dichotomy solution algorithm process for cost function 
In the next step, based on the optimal vector selected in the last step, 18 new voltage vectors, whose amplitude and phase angle are added or subtracted by half of last step optimal voltage vector, are selected to solve the cost function. Therefore, the search area decreases to half of the last step. After 14 steps iteration, the optimal voltage vector is selected as the reference vector for PWM modulator.

\section{FPGA-IN-THE-LOOP TEST RESULTS}

For the FPGA-in-the-Loop test, the plants including power converters, PMLSM and mechanical load are simulated in the MATLAB/Simulink in the computer. The control algorithm which is described as VHDL is working in the real FPGA board, for instance, Altera DE2-115 development board is used in this work. When the test is working, both the plants and control algorithm are working in real time connected with each other through Ethernet communication. Using this test method, the control algorithm can be completely tested as tested in the real test bench. The researchers do not need to pay too much time on hardware system. Therefore, the development time of control algorithms could be dramatically decreased. The whole FPGA-in-the-Loop test platform is described in Fig. 5. The parameters of PMLSM is shown in table I.

Fig. 6 presents the FPGA-in-the-Loop test results of both control methods. For the test, the speed is set to $125 \mathrm{rad} / \mathrm{s}$, the thrust load is set to $190 \mathrm{~N}$ and $380 \mathrm{~N}$ before and after $0.1 \mathrm{~S}$. The sampling frequency of conventional FCS-MPC is $40 \mathrm{kHz}$, which can insure the maximum switching frequency is no more than $20 \mathrm{kHz}$. The fixed switching frequency is $10 \mathrm{kHz}$ for the proposed CSF-MPC for PMLSM. It is clear to see that conventional FCS-MPC for PMLSM has larger current ripple compared with CSF-MPC. The current performance of CSF-MPC for PMLSM is elegant.
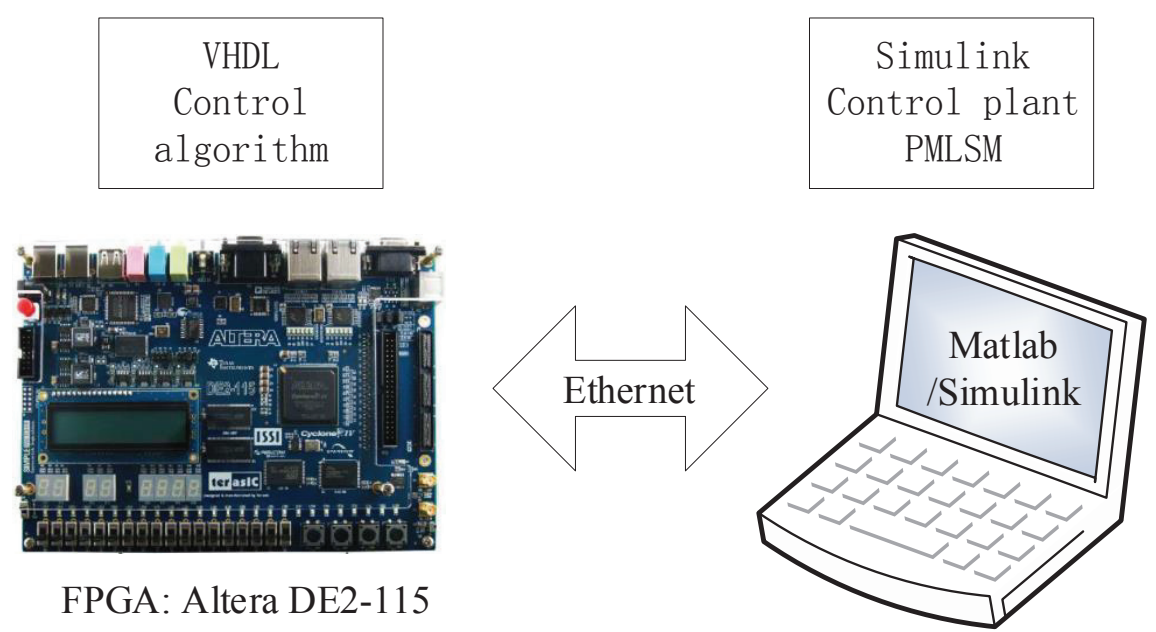

FPGA: Altera DE2-115

Fig. 5 Overview of FPGA-in-the-Loop test platform 
Table 1. Parameters of PMLSM

\begin{tabular}{|l|c|c|}
\hline \multicolumn{1}{|c|}{ Parameter } & Value & Unit of measurement \\
\hline Rated power & 1050 & $\mathrm{~W}$ \\
\hline Maximum power & 2030 & $\mathrm{~W}$ \\
\hline Current effective value & 4.6 & A rms \\
\hline Maximum current effective value & 24.6 & A rms \\
\hline Resistance & 3.1 & $\Omega$ \\
\hline Capactance & 32.2 & $\mathrm{mH}$ \\
\hline Back EMF constant & 26 & $\mathrm{Vrms} /(\mathrm{m} / \mathrm{s})$ \\
\hline Maximum speed & 3 & $\mathrm{~m} / \mathrm{s}$ \\
\hline Maximum acceration & 50 & $\mathrm{~m} / \mathrm{s} 2$ \\
\hline Continuous thrust & 180 & $\mathrm{~mm}$ \\
\hline Pole pitch $(2 \tau)$ & 32 & $\mathrm{~m}$ \\
\hline
\end{tabular}

(a)

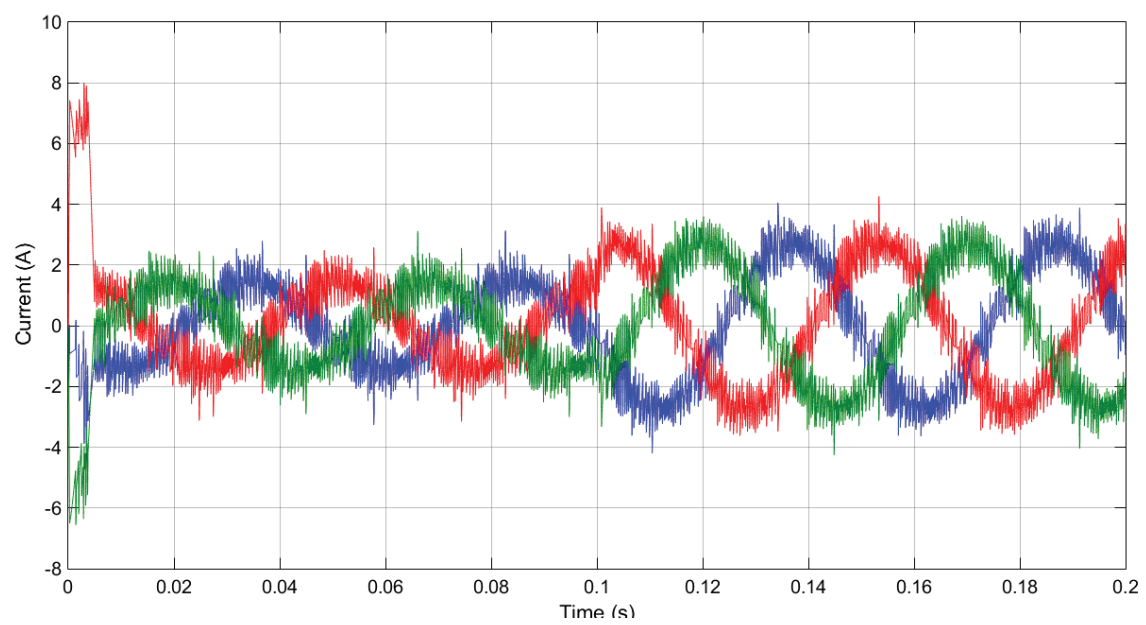

(b)

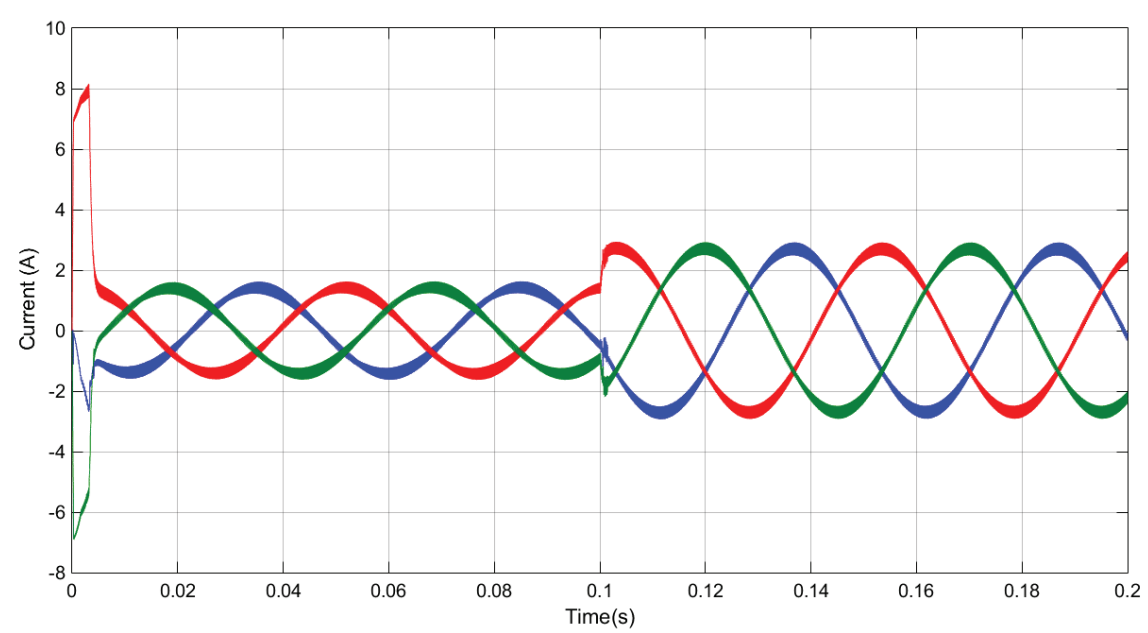

Fig. 6. FPGA-in-the-Loop test results comparison:

(a) conventional FCS-MPC; (b) proposed CSF-MPC 


\section{CONCLUSION}

CSF-MPC which is based on current prediction and control is proposed for PMLSM drive system. D and q axes currents are the control target in the control algorithm. Due to the effective dichotomy solution algorithm, the required optimal voltage vector can be selected on the whole voltage vector plane. So PWM modulator can be combined with conventional FCS-MPC method to generate the optimal voltage vector. This is reason why current ripple is dramatically reduced. With the novel FPGA-in-the-Loop test method, the effectiveness of proposed CSFMPC of PMLSM is proved and the test results are rather excited. The next step of this work will improve the algorithm further and test it on the real test bench.

\section{ACKNOWLEDGEMENTS}

This work was supported by the National Key Technology R\&D Program of China (2016YFB1200602-02), Shanghai science and technology innovation action plan (17511102302) and Hunan provincial science and technology major project, "medium and low speed maglev train integrated technology engineering and high reliability operation demonstration" (Porject No.: 2015GK1001).

\section{References}

1. Boldea I, Tutelea LN, Xu W, Pucci M. Linear Electric Machines, Drives, and MAGLEVs: An Overview. IEEE Transactions on Industrial Electronics. 2018;65(9):7504-15. doi: 10.1109/TIE.2017.2733492

2. Chen MY, Lu JS. High-precision motion control for a linear permanent magnet iron core synchronous motor drive in position platform. IEEE Transactions on Industrial Informatics. 2014;10(1):99-108. doi: 10.1109/TII.2013.2247044

3. Wang M, Li L, Pan D, Tang Y, Guo Q. High-bandwidth and strong robust current regulation for PMLSM drives considering thrust ripple. IEEE Transactions on Power Electronics. 2016;31(9):6646-57. doi: 10.1109/TPEL.2015.2499303

4. Vazquez S, Leon JI, Franquelo LG, Rodriguez J, Young HA, Marquez A, Zanchetta P. Model predictive control: A review of its applications in power electronics. IEEE Industrial Electronics Journal. 2014;8(1):16-31. doi: 10.1109/MIE.2013.2290138

5. Kouro S, Cortés P, Vargas R, Ammann U, Rodríguez J. Model predictive control - A simple and powerful method to control power converters. IEEE Transactions on industrial electronics. 2009;56(6):1826-38. doi: 10.1109/TIE.2008.2008349

6. Kouro S, Perez MA, Rodriguez J, Llor AM, Young HA. Model predictive control: MPC's role in the evolution of power electronics. IEEE Industrial Electronics Journal. 2015;9(4):8-21. doi: 10.1109/MIE.2015.2478920

7. Bordons C, Montero C. Basic principles of MPC for power converters: Bridging the gap between theory and practice. IEEE Industrial Electronics Journal. 2015;9(3):31-43. doi: 10.1109/MIE.2014.2356600 
8. Geyer T, Quevedo DE. Performance of multistep finite control set model predictive control for power electronics. IEEE Transactions on power electronics. 2015;30(3):1633-44. doi: 10.1109/TPEL.2014.2316173

9. Pacas M, Weber J. Predictive direct torque control for the PM synchronous machine. IEEE Transactions on industrial electronics. 2005;52(5):1350-6. doi: 10.1109/ TIE.2005.855662

10. Landsmann P, Stolze P, Kennel R. Optimal switching time calculation in predictive torque control. InPower Electronics and ECCE Asia (ICPE \& ECCE), 2011 IEEE 8th International Conference on 2011 May 30, p. 923-930. IEEE. doi: 10.1109/ICPE.2011.5944645

11. Zhang Y, Xu D, Liu J, Gao S, Xu W. Performance improvement of model-predictive current control of permanent magnet synchronous motor drives. IEEE Transactions on Industry Applications. 2017;53(4):3683-95. doi: 10.1109/TIA.2017.2690998

12. Ma Z, Saeidi S, Kennel R. FPGA implementation of model predictive control with constant switching frequency for PMSM drives. IEEE Transactions on Industrial Informatics. 2014;10(4):2055-63. doi: 10.1109/TII.2014.2344432

13. Nalakath S, Sun Y, Preindl M, Emadi A. Optimization-based Position Sensorless Finite Control Set Model Predictive Control for IPMSMs. IEEE Transactions on Power Electronics. 2017 Dec 19. doi: 10.1109/TPEL.2017.2784816

Information about the authors:

Liao Zhiming, M.E., Senior Engineering;

ORCID: 0000-0002-6005-4858;

E-mail: liaozhiming@tongji.edu.cn

Ma Zhixun, PhD, Associate Professor;

ORCID: 0000-0001-6772-0756;

E-mail: zhixun.ma@tongji.edu.cn

Zhao Yuanzhe, $\mathrm{PhD}$, Post Doctor;

ORCID: 0000-0002-9669-8209;

E-mail: zhaoyuanzhe@tongji.edu.cn

Sun Yan, PhD, Post Doctor;

ORCID: 0000-0003-4324-5553;

E-mail:17310079@tongji.edu.cn

Lin Guobin, M.E., Professor;

ORCID: 0000-0002-6583-4896;

E-mail: linguobin@tongji.edu.cn

\section{To cite this article:}

Ma Z, Zhao Y, Sun Y, et al. Constant Switching Frequency Model Predictive Control for Permanent Magnet Linear Synchronous Motor. Transportation Systems and Technology. 2018;4(3 suppl. 1): 279-288. doi: 10.17816/ transsyst201843s1279-288 